\title{
Self-Oscillating Gel as Novel Biomimetic Materials
}

\author{
Ryo Yoshida \\ Department of Materials Engineering, Graduate School of Engineering \\ The University of Tokyo, \\ Japan
}

\section{Introduction}

Polymer gel is a research field of polymer science which has made rapid progress during the past 20-30 years. Since the discovery of "volume phase transition" phenomena (Tanaka, 1978) as a turning point, many researchers have developed several kinds of stimuliresponsive polymer gels that exhibit reversible swelling-deswelling change in response to environmental changes such as solvent composition, temperature, $\mathrm{pH}$ change, etc. Especially, from the late 1980's or early 1990's, new functional gels which sense an external signal (sensor function), judge it (processor function) and take action (actuator function), have been developed as "intelligent gels" or "smart gels", and their applications have been demonstrated. As typical examples, many biomedical applications to actuator (artificial muscle), drug delivery systems (DDS), tissue engineering, purification or separation systems, biosensor, shape memory materials, molecular recognition systems, etc., have been extensively studied (Yoshida, 2005; Ottenbrite, et al., 2010; Miyata, 2002; Osada \& Khokhlov, 2002). And also, the gels has used in the field of micromachines and nanotechnology $[9,10]$. In addition to new synthetic methods to give unique functions by molecular design in nanoorder scale including supramolecular design, the design and construction of micro or nano material systems with the biomimetic functions have been attempted.

One of the characteristic behavior in living systems is autonomous oscillation, that is, spontaneous changes with temporal periodicity (called "temporal structure") such as heartbeat, brain waves, pulsatile secretion of hormone, cell cycle, and biorhythm. Although several stimuli-responsive polymer systems have been studied from the standpoint of biomimetics, polymer systems undergoing self-oscillation under constant condition without any on-off switching of external stimuli are still undeveloped. If autonomous polymer systems resembling living organisms can be realized by using completely synthetic polymers, unprecedented biomimetic materials may be created.

The author developed a novel polymer gel that cause autonomous mechanical oscillation without an external control in a completely closed solution. In order to realize the autonomous polymer system, the Belousov-Zhabotinsky (BZ) reaction, which is well-known for exhibiting temporal and spatiotemporal oscillating phenomena (Field, et al., 1985; Epstein \& Pojman, 1998), was focused. The BZ reaction is often analogically compared with the TCA cycle which is a key metabolic process taking place in the living body, and it is recognized as a chemical model for understanding several autonomous phenomena in 
biological systems. The overall process of the BZ reaction is the oxidation of an organic substrate, such as malonic acid (MA) or citric acid, by an oxidizing agent (bromate ion) in the presence of a strong acid and a metal catalyst. In the course of the reaction, the catalyst undergoes spontaneous redox oscillation. When the solution is homogeneously stirred, the color of the solution periodically changes, like a neon sign, based on the redox changes of the metal catalyst. When the solution is placed as a thin film in stationary conditions, concentric or spiral wave patterns develop in the solution. The wave of oxidized state propagating in the medium at a constant speed is called a "chemical wave". The significance of the BZ reaction has been recognized as a chemical model for understanding some aspects of biological phenomena, such as glycolytic oscillations or biorhythms, cardiac fibrillation, self-organization of amoeba cells, pattern formation on animal skin, visual pattern processing on retina, etc.

The author attempted to convert the chemical oscillation of the BZ reaction into a mechanical change in gels and generate an autonomous swelling-deswelling oscillation under non-oscillatory outer conditions. A copolymer gel consisting of $\mathrm{N}$ isopropylacrylamide (NIPAAm) and ruthenium tris(2,2'-bipyridine) ( $\left.\mathrm{Ru}(\mathrm{bpy})_{3}\right)$ was prepared. $\mathrm{Ru}(\mathrm{bpy})_{3}$, acting as a catalyst for the $\mathrm{BZ}$ reaction, is pendent to the polymer chains of NIPAAm. Poly(NIPAAm) is a well known thermosensitive polymer which exhibits a lower critical solution temperature (LCST) of approximately $32^{\circ} \mathrm{C}$, and the homopolymer gel undergoes a volume phase transition at that temperature. For the poly(NIPAAm-Co-Ru(bpy) 3 ) gel, the oxidation of $\mathrm{Ru}(\mathrm{bpy}) 3^{2+}$ moiety caused not only an increase in the swelling degree of the gel, but also a rise in the volume phase transition temperature. As a result, it is expected that the gel undergoes a cyclic swelling-deswelling change when the $\mathrm{Ru}(\mathrm{bpy})_{3}$ moiety is periodically oxidized and reduced under constant temperature. When the gel is immersed in an aqueous solution containing the substrates of the $\mathrm{BZ}$ reaction (malonic acid, nitric acid, and sodium bromate) except for the catalyst, the substrates penetrates into the polymer network and the BZ reaction occurs in the gel. Consequently, periodic redox changes induced by the $\mathrm{BZ}$ reaction produce periodic swelling-deswelling changes in the gel (Fugure 1).

Since being first reported in 1996 as a "self-oscillating gel" (Yoshida et al., 1996; 1997), the author have been systematically studying the self-oscillating polymer and gel as well as their applications to biomimetic or smart materials (Figure 2). In fact, as applications to autonomic biomimetic actuators, ciliary motion actuators (Tabata et al., 2002, 2003) and selfwalking gels (Maeda et al. 2007), etc. were realized. As an autonomic microconveyor, mass transport surface by utilizing peristaltic motion of the self-oscillating gel was also designed (Murase et al., 2009, 2010). In the case of the uncrosslinked linear polymer, the polymer undergoes spontaneous cyclic soluble-insoluble changes and the transmittance of the polymer solution oscillates autonomously (Yoshida et al., 2002a). In addition, submicronsized self-oscillating microgel beads were prepared by a precipitation polymerization method (Suzuki et al., 2008, 2009; Suzuki \& Yoshida, 2008a, 2008b, 2010; Taniguchi et al., 2010). In order to realize nano-actuators that exhibit autonomous oscillation on a nanometer scale (nano-oscillator) by utilizing the linear polymer chain or the microgels, their oscillating behavior were investigated through the optical transmittance or viscosity changes (Suzuki et al., 2009; Taniguchi et al., 2010; Hara \& Yoshida, 2008) of the polymer solution or microgel dispersions. 

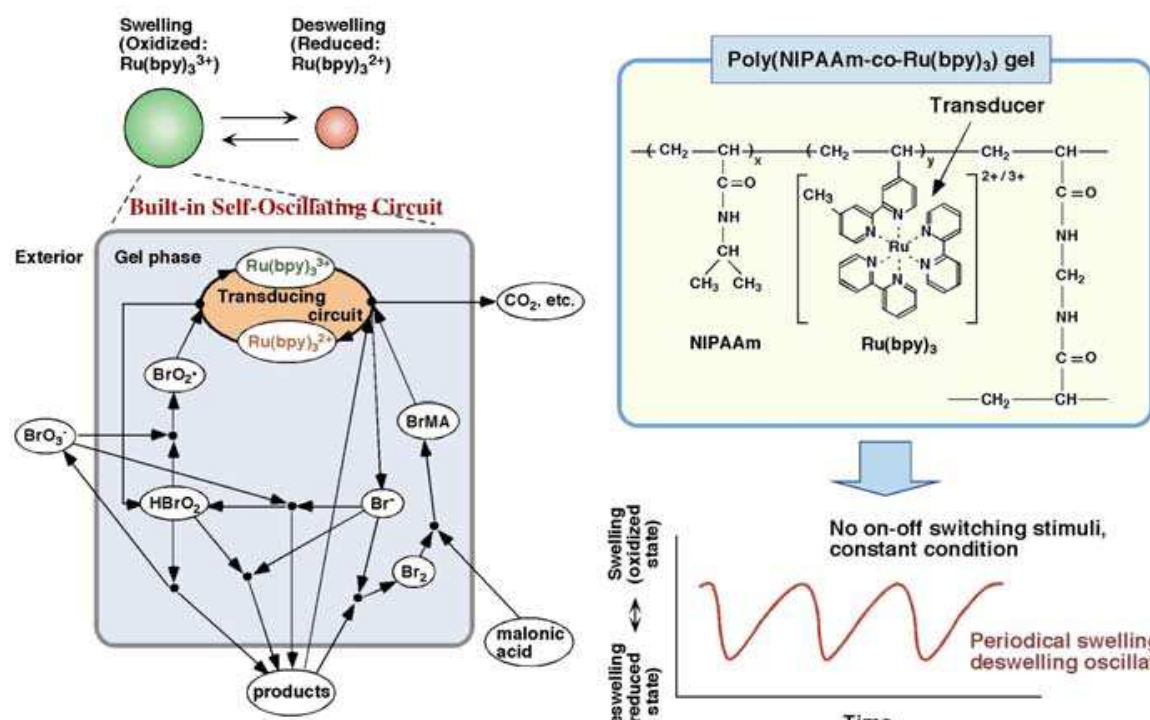

Reaction network of the $\mathrm{BZ}$ reaction

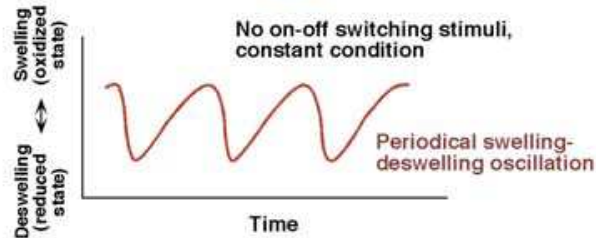
(FKN mechanism)

Fig. 1. Mechanism of self-oscillation for poly(NIPAAm-co-Ru(bpy) $3^{2+}$ ) gel coupled with the Belousov-Zhabotinsky reaction.

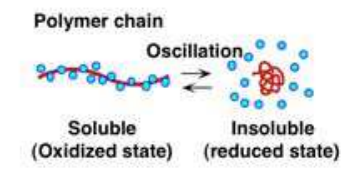

Self-oscillation of polymer chains
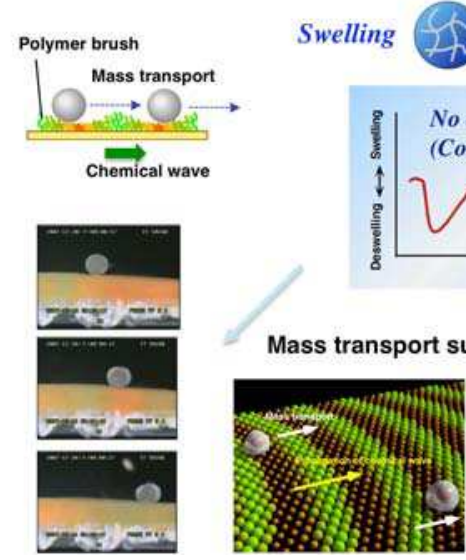

\section{- Viscosity oscillation}

\section{Self-flocculating/} dispersing oscillation of microgels

\section{Self-oscillating gel}

\section{Swelling

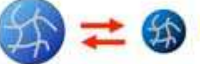 \\ Deswelling}

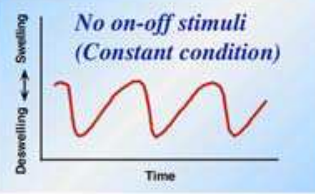

Peristaltic motion

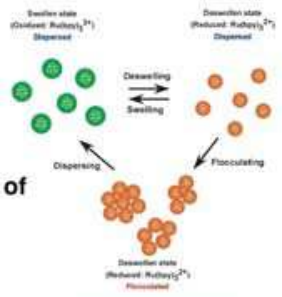

Fig. 2. Development of self-oscillating polymers and gels.

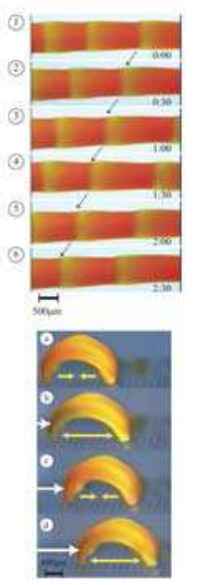




\section{Self-oscillating behavior of the gel}

\subsection{Self-oscillation of gel with smaller size than chemical wavelength and control of oscillating behavior}

Figure 3 shows the observed oscillating behavior under a microscope for the small cubic poly(NIPAAm-Co-Ru(bpy) $3^{2+}$ ) gel (each length of about $0.5 \mathrm{~mm}$ ). In miniature gels sufficiently smaller than the wavelength of the chemical wave (typically several $\mathrm{mm}$ ), the redox change of ruthenium catalyst can be regarded to occur homogeneously without pattern formation (Yoshida et al., 2000). Due to the redox oscillation of the immobilized $\mathrm{Ru}(\mathrm{bpy}) 3^{2+}$, mechanical swelling-deswelling oscillation of the gel autonomously occurs with the same period as for the redox oscillation. The volume change is isotropic and the gel beats as a whole, like a heart muscle cell. The chemical and mechanical oscillations are synchronized without a phase difference (i.e., the gel exhibits swelling during the oxidized state and deswelling during the reduced state).

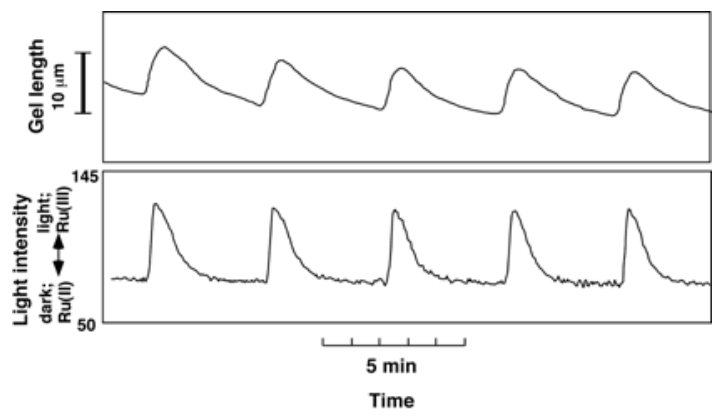

Fig. 3. Periodic redox changes of the miniature cubic poly(NIPAAm-co-Ru(bpy) $3^{2+}$ ) gel (lower) and the swelling-deswelling oscillation (upper) at $20^{\circ} \mathrm{C}$. Color changes of the gel accompanied by redox oscillations (orange: reduced state, light green: the oxidized state) were converted to 8-bit grayscale changes (dark: reduced, light: oxidized) by image processing. Transmitted light intensity is expressed as an 8-bit grayscale value. Outer solution: $[\mathrm{MA}]=62.5 \mathrm{mM} ;\left[\mathrm{NaBrO}_{3}\right]=84 \mathrm{mM} ;\left[\mathrm{HNO}_{3}\right]=0.6 \mathrm{M}$. (Yoshida et al., 2000)

Typically, the oscillation period increases with a decrease in the initial concentration of substrates. Further, in general, the oscillation frequency (the reciprocal of the period) of the $\mathrm{BZ}$ reaction tends to increase as the temperature increases, in accordance with the Arrhenius equation. The swelling-deswelling amplitude of the gel increases with an increase in the oscillation period and amplitude of the redox changes. Therefore the swelling-deswelling amplitude of the gel is controllable by changing the initial concentration of substrates as well as temperature.

As inherent behavior of the BZ reaction, the abrupt transition from steady state (nonoscillating state) to oscillating state occurs with a change in controlling parameter such as chemical composition, light, etc. By utilizing these characteristics, reversible on-off regulation of self-beating triggered by addition and removal of MA was successfully achieved (Yoshida et al., 2003). We showed the oscillating behavior of the gel when the stepwise concentration change in MA was repeated between lower concentration $(10 \mathrm{mM})$ in steady state and higher concentration $(25 \mathrm{mM})$ in oscillating state. At $10 \mathrm{mM}$, the redox oscillation does not occur and consequently the gel exhibited no swelling-deswellng 
changes. Then the concentration was quickly increased to $25 \mathrm{mM}$. Immediately after increasing concentration, the gel started self-beating. The beating stopped again as soon as the concentration was decreased back to the initial value. In these ways, reversible on-off regulation of self-beating triggered by MA was successfully achieved. Since there are some organic acid which can be the substrate for the BZ reaction (e.g., citric acid), the same regulation of beating is possible by using those organic acid instead of MA. As the gel has thermosensitivity due to the NIPAAm component, the beating rhythm can be also controlled by temperature. By utilizing the thermosensitivity, on-off control of the selfoscillation is also possible. At lower temperature, the oscillation does not occur because there is no remarkable difference in hydrophilicity of the polymer between the redox states, but the difference becomes remarkable at higher temperature and cause self-oscillation (Ito et al., 2003).

It is well known that the period of oscillation is affected by light illumination for the $\mathrm{Ru}(\mathrm{bpy}) 3^{2+}$-catalysed BZ reaction. Therefore we can intentionally make a pacemaker with a desired period (or wavelength) by local illumination of laser beam to the gel, or we can change the period (or wavelength) by local illumination to a pacemaker which already exists in the gel (Yoshida et al., 2002b). Optical on-off control of the self-oscillating motion of the gel were demonstrated (Takeoka et al., 2003; Shinohara et al., 2008a, 2008b).

\subsection{Peristaltic motion of gels with propagation of chemical wave}

When the gel size is larger than chemical wavelength, the chemical wave propagates in the gel by coupling with diffusion of intermediates. Then peristaltic motion of the gel is created. Figure 4 shows the cylindrical gel which is immersed in an aqueous solution containing the three reactants of the $\mathrm{BZ}$ reaction. The chemical waves propagate in the gel at a constant speed in the direction of the gel length (Maeda et al., 2008a). Considering the orange ( $\mathrm{Ru}(\mathrm{II})$ ) and green $(\mathrm{Ru}(\mathrm{III}))$ zones represent simply the shrunken and swollen parts respectively, the locally swollen and shrunken parts move with the chemical wave, like the peristaltic motion of living worms.

The tensile force of the cylindrical poly(NIPAAm-co-Ru(bpy) $3^{2+}$ ) gel with oscillation was also measured (Sasaki et al., 2003). Further, swelling behavior of the gel during selfoscillation was observed using CCD camera and the force due to the BZ reaction was evaluated using semiconductor gauge (Aoki et al., 2007). Self-oscillating swelling-deswelling behavior in samples with different swelling ratio was evaluated by this apparatus, and mechanical parameters such as Young's modulus, stress amplitude and oscillating period were quantitatively estimated. Dependency of initial length in samples on Young's modulus seems to be related to the propagation of chemical wave in the samples.

\section{Design of biomimetic actuator using self-oscillating polymer and gel}

\subsection{Ciliary motion actuator using self-oscillating gel}

Recently, microfabrication technologies such as photolithography are also attempted for preparation of microgels. Since any shape of gel can be created by these methods, application as a new manufacturing method for soft microactuator, microgel valve, gel display, etc. is expected. Microfabrication of gel has also been attempted by photolithography for application to such micro-devices (Yoshida et al., 2006a, 2006b). 


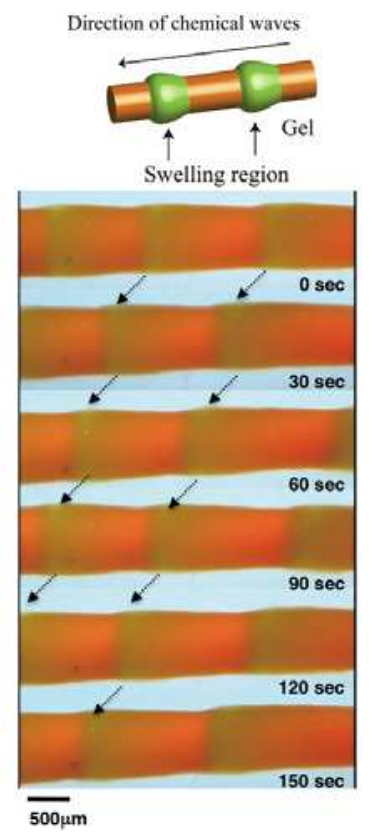

Fig. 4. Time course of peristaltic motion of poly(NIPAAm-co-Ru(bpy) $3^{2+}$-co-AMPS) gel in a solution of the $\mathrm{BZ}$ substrates at $18^{\circ} \mathrm{C}$. The green and orange colors correspond to the oxidized and reduced states of the Ru moiety in the gel, respectively (Maeda et al., 2008).

One of the promising fields of the MEMS is micro actuator array or distributed actuator systems. The actuators, which have a very simple actuation motion such as up and down motion, are arranged in an array form. If their motions are random, no work is extracted from this array. However, by controlling them to operate in a certain order, they can generate work as a system. A typical example of this kind of actuation array is a ciliary motion micro actuator array. There have been many reports on this system. Although various actuation principles have been proposed, all the previous work is based on the concept that the motion of actuators is controlled by external signals. If a self-oscillating gel plate with a micro projection structure array on top were realized, it would be expected that the chemical wave propagation would create dynamic rhythmic motion of the structure array. This proposed structure could exhibit spontaneous dynamic propagating oscillation producing a ciliary motion array (Figure 5)(Tabata et al., 2002, 2003).

A gel plate with micro projection array was fabricated by molding. First, moving mask deep-X-ray lithography was utilized to fabricate a PMMA plate with a truncated conical shape microstructure array. This step was followed by evaporation of a Au seed layer and subsequent electroplating of nickel to form the metal mold structure. Then, a PDMS mold structure was duplicated from the Ni structure and utilized for gel molding. The formation of gel was carried out by vacuum injection molding. A structure with a height of $300 \mu \mathrm{m}$ and bottom diameter of $100 \mu \mathrm{m}$ was successfully fabricated by the described process. The propagation of chemical reaction wave and dynamic rhythmic motion of the micro projection array were confirmed by chemical wave observation and displacement measurements. Motion of the top with $5 \mu \mathrm{m}$ range in both lateral and vertical directions, 
and elliptical motion of the projection top were observed. The feasibility of the new concept of the ciliary motion actuator made of self-oscillating polymer gel was successfully confirmed. The actuator may serve as a micro-conveyer to transport objects on the surface.

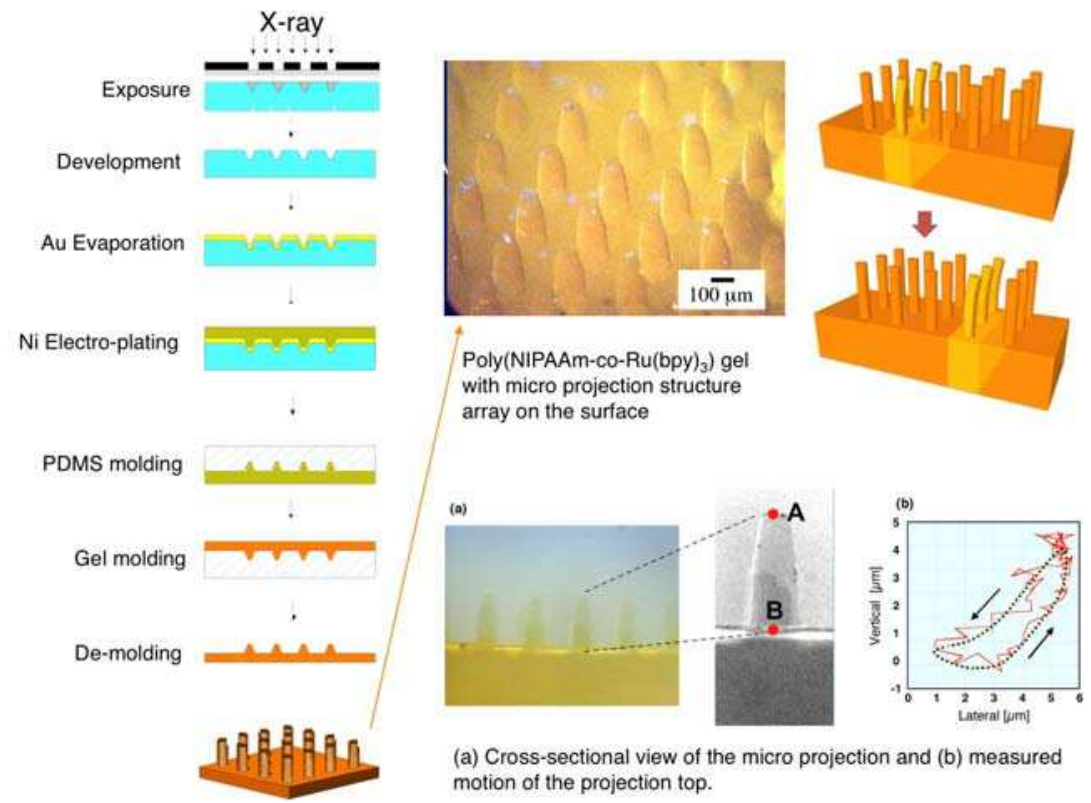

Fig. 5. Fabrication of ciliary motion actuator (artificial cilia) using self-oscillating gel (Tabata et al., 2002, 2003).

\subsection{Self-walking gel}

Further, we successfully developed a novel biomimetic walking-gel actuator made of selfoscillating gel (Maeda et al., 2007). To produce directional movement of gel, asymmetrical swelling-deswelling is desired. For these purposes, as a third component, hydrophilic 2acrylamido-2'-methylpropanesulfonic acid (AMPS) was copolymerized into the polymer to lubricate the gel and to cause anisotropic contraction. During polymerization, the monomer solution faces two different surfaces of plates; a hydrophilic glass surface and a hydrophobic Teflon surface. As the thickness of the spacer is thin $(0.5 \mathrm{~mm})$, the surface property of the plate may affect the distribution of the monomer in the solution. Since $\mathrm{Ru}(\mathrm{bpy}) 3^{2+}$ monomer is hydrophobic, it easily migrates to the Teflon surface side. As a result, a non-uniform distribution along the height is formed by the components, and the resulting gel has gradient distribution for the content of each component in the polymer network.

In order to convert the bending and stretching changes to one-directional motion, we employed a ratchet mechanism. A ratchet base with an asymmetrical surface structure was fabricated. On the ratchet base, the gel repeatedly bends and stretches autonomously resulting in the forward motion of the gel, while sliding backwards is prevented by the teeth of the ratchet. Figure 6 shows successive profiles of the "self-walking" motion of the gel like a looper in the BZ substrate solution under constant temperature. The walking velocity of the gel actuator was approximately $170 \mu \mathrm{m} / \mathrm{min}$. Since the oscillating period and the 
propagating velocity of chemical wave change with concentration of substrates in the outer solution, the walking velocity of the gel can be controlled. By using the gel with gradient structure, other type of actuator which generates a pendulum motion is also realized (Maeda et al., 2008b).

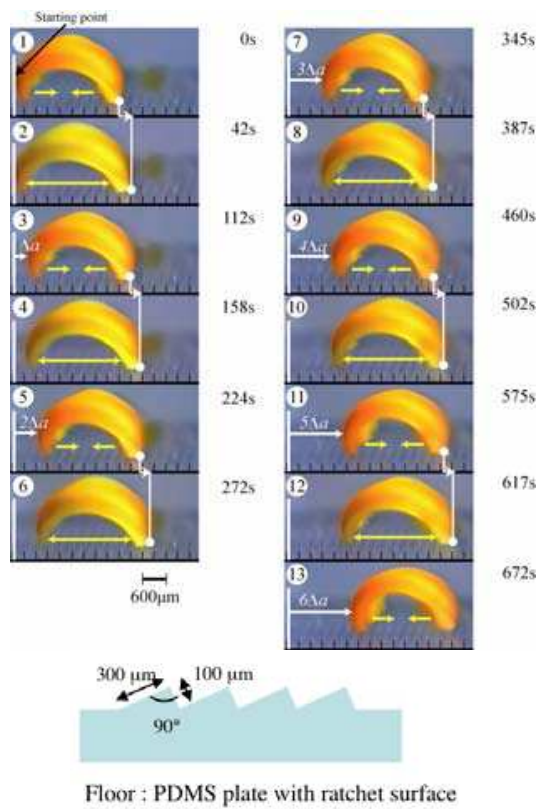

Fig. 6. Time course of self-walking motion of the gel actuator. During stretching, the front edge can slide forward on the base, but the rear edge is prevented from sliding backwards. Oppositely, during bending, the front edge is prevented from sliding backwards while the rear edge can slide forward. This action is repeated, and as a result, the gel walks forward. Outer solution: $[\mathrm{MA}]=62.5 \mathrm{mM},\left[\mathrm{NaBrO}_{3}\right]=84 \mathrm{mM},\left[\mathrm{HNO}_{3}\right]=0.894 \mathrm{M}, 18^{\circ} \mathrm{C}$ (Maeda et al., 2007).

\subsection{Mass transport surface utilizing peristaltic motion of gel}

Further, we attempted to transport an object by utilizing the peristaltic motion of the selfoscillating gel (Murase et al., 2009a, 2009b, 2010). In order to control the transportability, it is necessary to enhance the driving force as a conveyer. It is effective to copolymerize AMPS to poly(NIPAAm-co-Ru(bpy) $3^{2+}$ ) gel network to generate large amplitude of volume change of the self-oscillating gel. For the design of mass transport surface, the influence of the AMPS's feed ratio on the swelling-deswelling properties of poly(NIPAAm-co$\mathrm{Ru}(\mathrm{bpy}) 3^{2+}$-co-AMPS) gels and mass transportability of the gels were investigated.

The gel had a microphase-separated structure when the AMPS's feed ratio was less than $5 \mathrm{~mol}$ $\%$ due to the effect of the poor solvent in the polymerization process. On the other hand, when the AMPS's feed ratio is more than $10 \mathrm{~mol} \%$, the gel had a homogeneous structure. The microphase-separated structure highly improved the swelling-deswelling kinetics and generated swelling-deswelling amplitude more than $10 \%$ of the gel thickness, that was approximately 10 times larger than that of the gel with a homogeneous network structure. 
As a model object, a cylindrical poly(acrylamide) (PAAm) gel was put on the gel surface. It was observed that the PAAm gel was transported on the gel surface with the propagation of the chemical wave as it rolled (Figure 7) when the AMPS's feed ratio was low (less than 2.5 mol \%). It was found that the cylindrical PAAm gel was not transported if the inclination angle of the wave front was less than approximately 0.05 rad. The mass transportability does not depend on the velocity of the chemical wave but on the diameter of the cylindrical PAAm gel and the inclination angle of the wave front. We have proposed a model to describe the mass transport phenomena based on the Hertz contact theory, and the relation between the transportability and the peristaltic motion was investigated for the cases that the loaded gel cargos were gel bead and cylindrical gel (Murase et al., 2010). It was found that the surface design to form grooves was efficient to increase contact areas when the wave front of the peristaltic motion reaches the loaded cargo and useful to transport not only cylindrical cargos but also spherical cargos in desired directions.

The functional gel surface generating autonomous and periodic peristaltic motion has a potential for several new applications such as a conveyer to transport soft materials, a formation process for ordered structures of micro- and/or nanomaterials, a self-cleaning surface and so on.
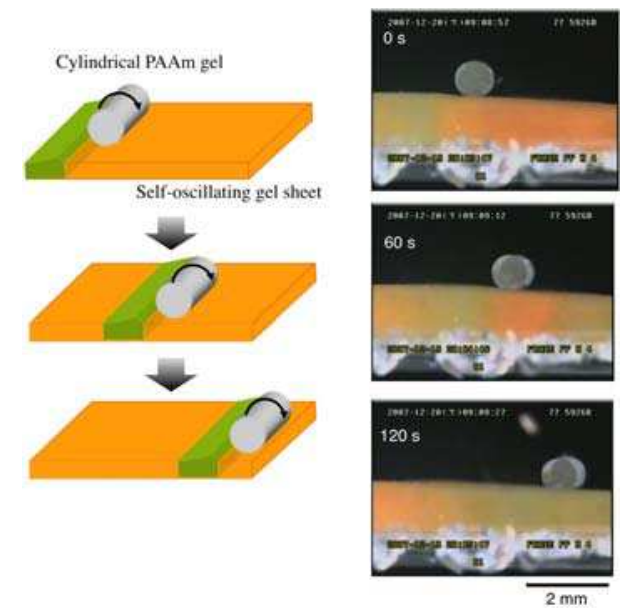

Fig. 7. Schematic illustration of mass transport on the peristaltic surface (left) and observed transport of cylindrical PAAm gel on the poly(NIPAAm-co-Ru(bpy) $3_{3}{ }^{2+}$-Co-AMPS) gel sheet in a solution of the $\mathrm{BZ}$ substrates $\left(\mathrm{MA}, \mathrm{NaBrO}_{3}\right.$ and $\left.\mathrm{HNO}_{3}\right)$ (right) (Murase et al., 2009).

\subsection{Control of chemical wave propagation through gap junction in self-oscillating gel array}

A chemomechanical actuator utilizing a reaction-diffusion wave across gap junction was constructed toward a novel mircoconveyer by micropatterned self-oscillating gel array (Tateyama et al., 2008). Unidirectional propagation of the chemical wave the BZ reaction was induced on gel arrays. In the case of using a triangle-shaped gel as an element of the array, the chemical wave propagated from the corner side of the triangle gel to the plane side of the other gel (C-to-P) across the gap junction, whereas it propagated from the plane side to the corner side (P-to-C) in the case of the pentagonal gel array. Numerical analysis 
based on theoretical model was done for understanding the mechanism of unidirectional propagation in triangle and pentagonal gel arrays. By fabricating different shapes of gel arrays, control of the direction is possible. The swelling and deswelling changes of the gels followed the unidirectional propagation of chemical wave.

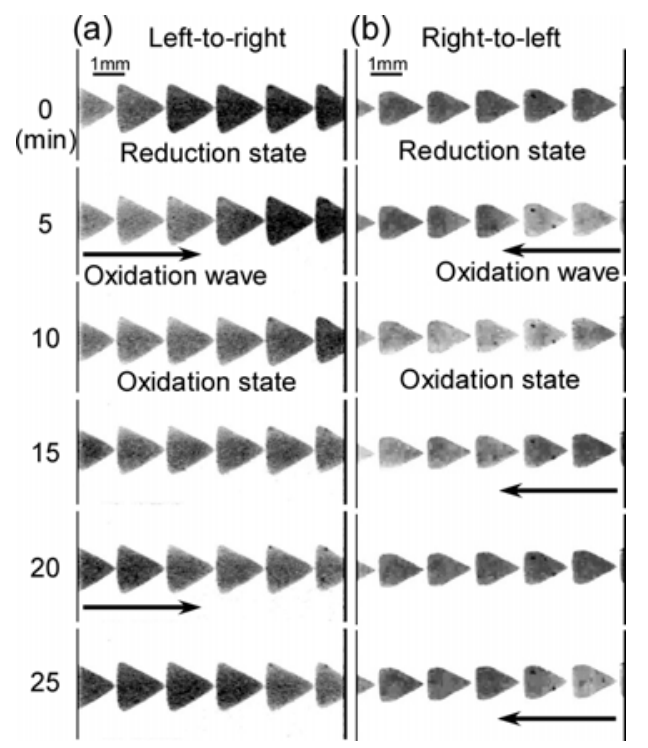

Fig. 8. Propagating behavior of the chemical wave on the (a) triangle gel array and (b) pentagonal gel array (Tateyama et al., 2008).

\section{Self-oscillating polymer chains and microgels as "nano-oscillator"}

\subsection{Self-oscillation of polymer chains with rhythmical soluble-insoluble changes}

In self-oscillating gel, redox changes of $\mathrm{Ru}(\mathrm{bpy}) 3^{2+}$ catalyst are converted to conformational changes of polymer chain by polymerization. The conformational changes are amplified to macroscopic swelling-deswelling changes of the polymer network by crosslinking. Further, when the gel size is larger than chemical wavelength, the chemical wave propagates in the gel by coupling with diffusion. Then peristaltic motion of the gel is created. In this manner, a synchronization process exists from microscopic to macroscopic level in the self-oscillating gel.

The periodic changes of linear and uncrosslinked polymer chains can be easily observed as cyclic transparent and opaque changes for the polymer solution with color changes due to the redox oscillation of the catalyst (Yoshida et al., 2002a). Figure 9(a) shows the oscillation profiles of transmittance for a polymer solution which consists of linear poly(NIPAAm-co$\mathrm{Ru}\left(\right.$ bpy) $\left.{ }_{3}^{2+}\right), \mathrm{MA}, \mathrm{NaBrO}_{3}$ and $\mathrm{HNO}_{3}$ at constant temperatures. The wavelength $(570 \mathrm{~nm})$ at the isosbestic point of reduced and oxidized states was used to detect the optical transmittance changes based on soluble-insoluble changes of the polymer, not on the redox changes of the $\mathrm{Ru}(\mathrm{bpy})_{3}$ moiety. Synchronized with the periodical changes between $\mathrm{Ru}(\mathrm{II})$ and $\mathrm{Ru}(\mathrm{III})$ states of the $\mathrm{Ru}(\mathrm{bpy}) 3^{2+}$ site, the polymer becomes hydrophobic and hydrophilic, and exhibits cyclic soluble-insoluble changes. 
By grafting the polymers or arraying the gel beads on the surface of substrates, we have attempted to design self-oscillating surfaces as nano-conveyers. The self-oscillating polymer was covalently immobilized on a glass surface and self-oscillation was directly observed at a molecular level by AFM (Ito et al., 2006). The self-oscillating polymer with $N$-succinimidyl group was immobilized on an aminosilane-coupled glass plate. While no oscillation was observed in pure water, nano-scale oscillation was observed in an aqueous solution containing the BZ substrates. The amplitude was about 10-15 $\mathrm{nm}$ and the period was about $70 \mathrm{sec}$, although some irregular behavior was observed due to no stirring. The amplitude was less than that in solution, as observed by DLS (23.9 and $59.6 \mathrm{~nm}$ ). This smaller amplitude may be because the structure of the immobilized polymer was a loop-train-tail: the moving regions were shorter than that of the soluble polymer. The amplitude and frequency were controlled by the concentration of reactant, as observed in the solution. Here nano-scale molecular self-oscillation was observed for the first time. The oscillation polymer chain may be used as a component of a nano-clock or nano-machine.

(a)

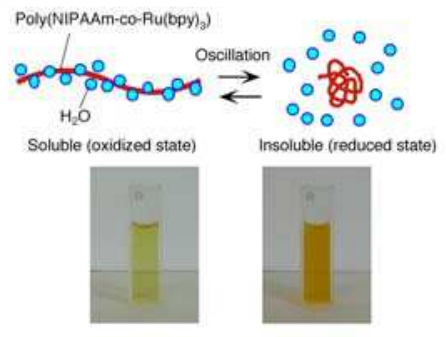

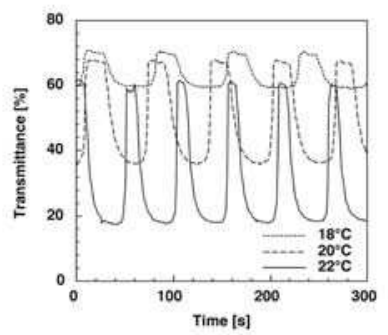

(b)

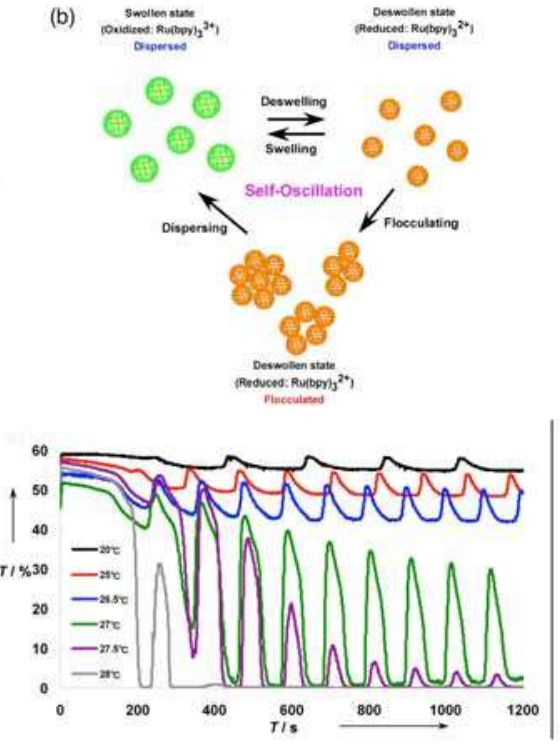

Fig. 9. (a) Oscillating profiles of optical transmittance for poly(NIPAAm-co-Ru(bpy) $3^{2+}$ ) solution at several temperatures (Yoshida et al., 2002). (b) Self-oscillating profiles of optical transmittance for microgel dispersions at several temperatures. The microgels were dispersed in aqueous solutions containing $\mathrm{MA}(62.5 \mathrm{mM}), \mathrm{NaBrO}_{3}(84 \mathrm{mM})$, and $\mathrm{HNO}_{3}$ (0.3M). Microgel concentration was $0.25 \mathrm{wt} \%$ (Suzuki et al, 2008).

\subsection{Self-flocculating/dispersing oscillation of microgels}

We then prepared submicron-sized poly(NIPAAm-co-Ru(bpy) $3^{2+}$ ) gel beads by surfactantfree aqueous precipitation polymerization, and analyzed the oscillating behavior (Suzuki et al., 2008, 2009; Suzuki \& Yoshida, 2008a, 2008b, 2010; Taniguchi et al., 2010; Sakai \& Yoshida, 2004). Figure 9(b) shows the oscillation profiles of transmittance for the microgel 
dispersions. At low temperatures $\left(20-26.5^{\circ} \mathrm{C}\right)$, on raising the temperature, the amplitude of the oscillation became larger. The increase in amplitude is due to increased deviation of the hydrodynamic diameter between the $\mathrm{Ru}(\mathrm{II})$ and $\mathrm{Ru}(\mathrm{III})$ states. Furthermore, a remarkable change in waveform was observed between 26.5 and $27{ }^{\circ} \mathrm{C}$. Then the amplitude of the oscillations dramatically decreased at $27.5^{\circ} \mathrm{C}$, and finally the periodic transmittance changes could no longer be observed at $28^{\circ} \mathrm{C}$. The sudden change in oscillation waveform should be related to the difference in colloidal stability between the $\mathrm{Ru}(\mathrm{II})$ and $\mathrm{Ru}(\mathrm{III})$ states. Here, the microgels should be flocculated due to lack of electrostatic repulsion when the microgels were deswollen. The remarkable change in waveform was only observed at higher dispersion concentrations (greater than $0.225 \mathrm{wt} . \%$ ). The self-oscillating property makes microgels more attractive for future developments such as microgel assembly, optical and rheological applications, etc.

\subsection{Viscosity oscillation of polymer solution and microgel dispersion}

In the case of the self-oscillating polymer solution or the mircogel dispersion, the solubility changes or the swelling-deswelling changes can be measured as viscosity changes as well as optical transmittance changes of the solution or the dispersion. Actually, we succeeded in observing the viscosity self-oscillation for the polymer solution induced by the BZ reaction at the constant temperature (Hara \& Yoshida, 2008). The viscosity self-oscillation was originated by the difference between solubilities of the polymer chain in the reduced and oxidized states. The effects of the polymer concentration and the temperature of the viscosity self-oscillation were investigated. As a result, the viscosity self-oscillating behavior significantly depended on the polymer concentration and the temperature of the polymer solution. The period of the viscosity self-oscillation decreased with increasing temperature in accordance with the Arrenius equation.

Recently, we have achieved autonomously oscillating viscosity in a microgel dispersion using autonomously oscillating microgels (Suzuki et al., 2009; Taniguchi et al., 2010). We found out that viscosity oscillation occurs in two different manners, exhibiting a simple pulsatile waveform or a complex waveform with two peaks per period (Figure 10(a)). It was suggested that the difference in waveform is due to the difference in oscillating manner of the microgels: swelling/deswelling or dispersing/flocculating oscillation as mentioned before. We can control rhythm and amplitude of the oscillation using these two phenomena of the microgels. In order to characterize the viscosity oscillation, two types of the microgels were synthesized by changing the feed ratio of $\mathrm{Ru}(\mathrm{bpy})_{3}$ and cross-linker. Viscosity of the microgel dispersions at high salt concentrations could be controlled by changing concentrations of the microgels. Autonomously oscillating viscosity was only measured when concentration of the microgels was high. The amplitude of the oscillation became bigger with increasing concentrations of the microgels. By adjusting the concentration of the substrates for the BZ reaction, we could achieve the constant oscillation for a long time. Moreover, with increasing $\mathrm{Ru}(\mathrm{bpy})_{3}$ and decreasing the cross-linker, microgels showed a high degree of swelling/deswelling oscillation, resulting in bigger amplitudes of autonomously oscillating viscosity (Figure 10(b)).

This technology could be applied in many applications as electro- or magnetic- rheological (ER or MR) fluids have been done. In particular, the dispersion with autonomously oscillating viscosity may be used as a micropump, which could realize novel microfluidics devices. 
(a)
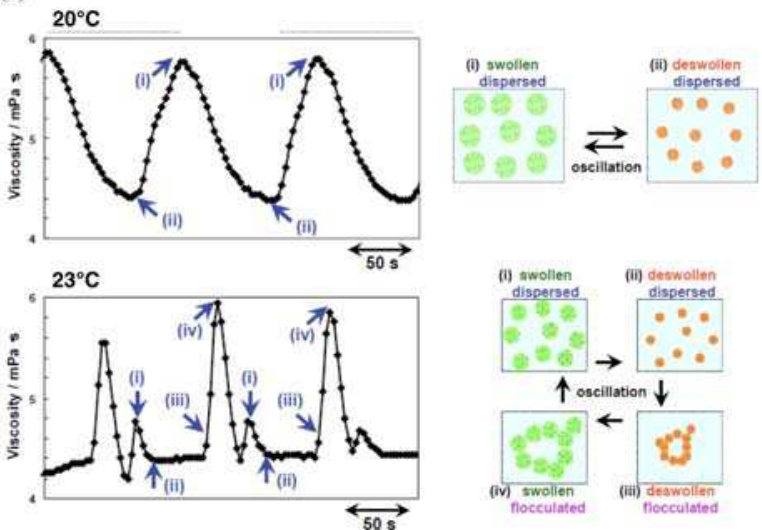

(b)

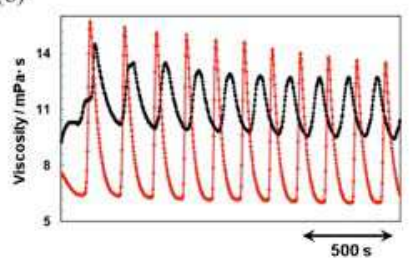

Fig. 10. (a) Two different types of oscillating waveforms observed at $20^{\circ} \mathrm{C}$ and $23^{\circ} \mathrm{C}$. The numbers in each oscillating profiles refer to the corresponding cartoons (Suzuki et al., 2009). (b) Autonomously oscillating profiles of viscosity in the microgel dispersions measured at $15^{\circ} \mathrm{C}$. for NIPAAm:Ru(bpy) $3: \mathrm{BIS}=95: 1: 4$ (black line) and 96:2:2 microgels (red line). The microgels $(5.0 \mathrm{wt} \%)$ were dispersed in aqueous solutions containing $\mathrm{MA}(100 \mathrm{mM}), \mathrm{NaBrO}_{3}$ $(150 \mathrm{mM})$, and $\mathrm{HNO}_{3}(500 \mathrm{mM})$. Both data were taken at constant shear rate of $132 \mathrm{~s}^{-1}$ (Taniguchi et al., 2010).

\subsection{Fabrication of microgel beads monolayer}

As discussed in the previous section, we have been interested in the construction of micro/nano-conveyers by grafting or arraying self-oscillating polymer or gel beads. For this purpose, a fabrication method for organized monolayers of microgel beads was investigated (Sakai et al., 2007). A 2D close-packed array of thermosensitive microgel beads was prepared by double template polymerization. First, a 2D colloidal crystal of silica beads with $10 \mathrm{~mm}$ diameter was obtained by solvent evaporation. This monolayer of colloidal crystal can serve as the first template for preparation of macroporous polystyrene. The macroporous polystyrene trapping the crystalline order can be used as a negative template for fabricating a gel bead array. By this double template polymerization method, functional surfaces using thermosensitive PNIPAAm gel beads were fabricated. It was observed that topography of the surface changed with temperature. The fabrication method demonstrated here was so versatile that any kind of gel beads could be obtained. This method may be a key technology to create new functional surface. Actually, monolayer of self-oscillating microgel beads was fabricated by this method and the chemical wave propagation on the monolayer was observed. 


\section{Attempts of self-oscillation under physiological conditions}

\subsection{Self-oscillation of polymer chains under acid-free conditions}

So far, the author had succeeded in developing a novel self-oscillating polymer (or gel) by utilizing the $\mathrm{BZ}$ reaction. However, the operating conditions for the self-oscillation are limited to conditions under which the BZ reaction occurs. For practical applications as functional bio- or biomimetic materials, it is necessary to design a self-oscillating polymer which acts under biological environments. To cause self-oscillation of polymer systems under physiological conditions, BZ substrates other than organic ones, such as malonic acid and citric acid, must be built into the polymer system itself. Therefore, we took the two steps; the first step is to design a novel self-oscillating polymer chains with incorporated $\mathrm{pH}$-control sites, that is, the polymer chains which exhibit rhythmic oscillations in an aqueous solutions containing only the two BZ substrates, without using acid as an added agent. For this purpose, 2-acrylamido-2-methylpropanesulfonic acid (AMPS) was incorporated into the poly(NIPAAm-co-Ru(bpy) $3^{2+}$ ) chain as the $\mathrm{pH}$ control site (Hara \& Yoshida, 2005a, 2005b).

The self-oscillating transmittance change for the solutions of poly(NIPAAm-co-Ru(bpy) $3^{2+}$ co-AMPS) $\left(20: 10: 70 \mathrm{wt} \%\right.$ in feed) at three constant temperatures $\left(18,21\right.$ and $\left.24^{\circ} \mathrm{C}\right)$ were demonstrated. Under acid-free conditions and in the presence of only two BZ substrates (malonic acid and sodium bromate), we succeeded in causing soluble-insoluble selfoscillation of a polymer solution. Oscillating behavior were remarkably influenced by the temperature, polymer concentration, and composition.

\subsection{Self-oscillation under oxidant-free conditions}

As the next step, we attempted to introduce the oxidizing agent into the polymer. Methacrylamidopropyltrimethylammonium chloride (MAPTAC), with a positively charged group, was incorporated into the poly(NIPAAm-co-Ru(bpy) $3^{2+}$ ) as a capture site for an anionic oxidizing agent (bromate ion) (Hara et al., 2005). The bromate ion was introduced into the MAPTAC-containing polymer through ion-exchange. Under the conditions in which only two BZ substrates (malonic acid and sulfuric acid) were present, solubleinsoluble self-oscillation of the polymer was observed.

In the self-oscillating polymer solution system induced by the BZ reaction, self-oscillation was achieved without addition of oxidizing agent by utilizing the MAPTAC-containing polymer which included sodium bromate as a counter ion. The self-oscillating behavior was controllable by temperature. The polymer has two advantages because of the higher LCST; one is self-oscillation around body temperature, and the other is oscillation for a longer time without intermolecular aggregation among the polymer chains in the reduced state.

\subsection{Self-oscillation under acid- and oxidant-free condition}

Further, we have synthesized a quarternary copolymer which includes both $\mathrm{pH}$-control and oxidant-supplying sites in the poly(NIPAAm-co-Ru(bpy) 3 ) chain at the same time (Hara \& Yoshida, 2008b). In the polymer, AMPS was incorporated as a $\mathrm{pH}$-control site, and MAPTAC with a positively charged group was incorporated as a capture site for an anionic oxidizing agent (bromate ion). By using the polymer, self-oscillation under biological conditions where only the organic acid (malonic acid) exists was actually achieved (Figure 11). Other than malonic acid, citric acid or malic acid, which is biorelated organic acid, can be a substrate of the BZ reaction. For practical medical use, however, it would be necessary 
to avoid exchange of toxic ion such as bromate to outside of the gel. In addition, working temperature must be improved. The design of gel for biomedical application is under investigation.
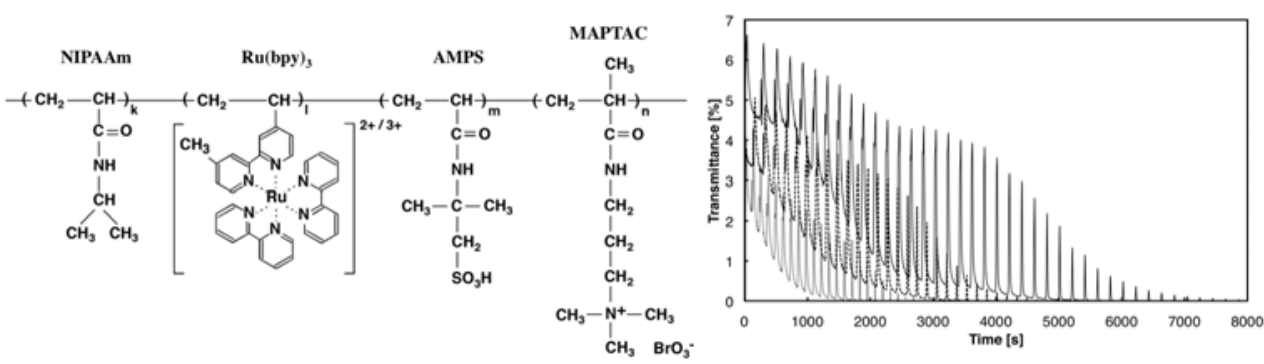

Fig. 11. Chemical structure of poly(NIPAAm-co-Ru(bpy) $3^{2+}$-co-AMPS-co-MAPTAC) (upper) and the oscillating profiles of the optical transmittance for the polymer solution at $12^{\circ} \mathrm{C}$ when only MA (0.7M: fine dotted line, $0.5 \mathrm{M}$ : rough dotted line, $0.3 \mathrm{M}$ : solid line) is added to the solution (lower) (Hara \& Yoshida, 2008).

\section{References}

Aoki, R., Enoki, M. and Yoshida, R. (2007). Key Eng. Mater. 353, 2235-2238.

Epstein, I.R., Pojman, J.A. (1998). An Introduction to Nonlinear Chemical Dynamics: Oscillations, Waves, Patterns, and Chaos, Oxford University Press, New York.

Field R.J.; Burger, M. Eds. (1985). Oscillations and Traveling Waves in Chemical Systems, John Wiley \& Sons, New York.

Hara, Y. and Yoshida, R. (2008). (a) J. Chem. Phys. 128, 224904. (b) J. Phys. Chem. B 112, 84278429.

Hara, Y. and Yoshida, R. (2005). (a) Langmuir 21, 9773-9776. (b) J. Phys. Chem. B 109, 94519454.

Hara, Y., Sakai, T., Maeda, S., Hashimoto, S. and Yoshida, R. (2005). J. Phys. Chem. B 109, 23316-23319.

Ito, Y., Nogawa, N. and Yoshida, R. (2003). Langmuir 19, 9577-9579.

Ito, Y., Hara, Y., Uetsuka, H., Hasuda, H., Onishi, H., Arakawa, H., Ikai, A. and Yoshida, R. (2006). J. Phys. Chem. B 110, 5170-5173.

Maeda, S., Hara, Y., Sakai, T., Yoshida, R. and Hashimoto, S. (2007). Adv. Mater. 19, 34803484.

Maeda, S., Hara, Y., Yoshida, R. and Hashimoto, S. (2008). (a) Angew. Chem. Int. Ed. 47, 66906693. (b) Macromol. Rapid Commun. 29, 401-405.

Miyata, T. (2002). Stimuli-responsive polymer and gels: in Supramolecular Design for Biological Applications, Yui, N. Ed.; CRC Press, Boca Raton, pp.191-225.

Murase, Y., Maeda, S., Hashimoto, S. and Yoshida, R. (2009a). Langmuir 25, 483-489.

Murase, Y., Takeshima, R. and Yoshida, R. (2009b). Trans. Mater. Res. Soc. Japan 34, 171-174 .

Murase, Y., Hidaka, M. and Yoshida, R. (2010). Sensors and Actuators B 149, 272-283.

Osada, Y., Khokhlov, A.R., Eds. (2002). Polymer Gels and Networks, Marcel Dekker, New York. 
Ottenbrite, R.M., Park, K., Okano, T. and Peppas, N.A. (Eds) (2010). Biomedical Applications of Hydrogels Handbook, Springer, New York.

Sakai, T. and Yoshida, R. (2004). Langmuir 20, 1036-1038.

Sakai, T., Takeoka, Y., Seki, T. and Yoshida, R. (2007). Langmuir 23, 8651-8654.

Sasaki, S., Koga, S., Yoshida, R. and Yamaguchi, T. (2003). Langmuir 19, 5595-5600.

Shinohara, S., Seki, T., Sakai, T., Yoshida, R. and Takeoka, Y. (2008). (a) Angew. Chem. Int. Ed. 47, 9039-9043. (b) Chem. Commun. 4735-4737.

Suzuki, D., Sakai, T. and Yoshida, R. (2008). Angew. Chem. Int. Ed. 47, 917-920.

Suzuki, D. and Yoshida, R. (2008). (a) J. Phys. Chem. B 112, 12618-12624. (b) Macromolecules $41,5830-5838$.

Suzuki, D., Taniguchi, H. and Yoshida, R. (2009). J. Am. Chem. Soc. 131, 12058-12059.

Suzuki, D. and Yoshida, R. (2010). Polym. J. 42, 501-508.

Tabata, O., Hirasawa, H., Aoki, S., Yoshida, R. and Kokufuta, E. (2002). Sensors and Actuators A 95, 234-238.

Tabata, O., Kojima, H., Kasatani, T., Isono, Y. and Yoshida, R. Chemo-mechanical actuator using self-oscillating gel for artificial cilia, Proceedings of the International Conference on MEMS 2003, pp.12-15 (2003).

Takeoka, Y., Watanabe, M. and Yoshida, R. J. Am. Chem. Soc. (2003). 125, 13320-13321.

Tanaka, T. Phys. Rev. Lett. 1978, 40, 820-823.

Taniguchi, H., Suzuki, D. and Yoshida, R. (2010). J. Phys. Chem. B 114, 2405-2410.

Tateyama, S., Shibuta, Y. and Yoshida, R. (2008). J. Phys. Chem. B 112, 1777-1782.

Yoshida, R., Takahashi, T., Yamaguchi, T. and Ichijo, H. (1996). J. Am. Chem. Soc. 118, 51345135 .

Yoshida, R., Takahashi, T., Yamaguchi, T. and Ichijo, H. (1997). Adv. Mater. 9, 175-178.

Yoshida, R., Tanaka, M., Onodera, S., Yamaguchi, T. and Kokufuta, E. (2000). J. Phys. Chem. A 104, 7549-7555.

Yoshida, R., Sakai, T., Ito, S. and Yamaguchi, T. (2002a). J. Am. Chem. Soc. 124, 8095-8098.

Yoshida, R., Sakai, T., Tabata, O. and Yamaguchi, T. (2002b). Sci. Tech. Adv. Mater. 3, 95-102.

Yoshida, R., Takei, K. and Yamaguchi, T. (2003). Macromolecules 36, 1759-1761.

Yoshida, R. (2005). Curr. Org. Chem. 9, 1617-1641.

Yoshida, R., Omata, K., Yamaura, K., Ebata, M., Tanaka, M., Takai, M. (2006a). Lab on a Chip 6, 1384-1386.

Yoshida, R., Omata, K., Yamaura, K., Sakai, T., Hara, Y., Maeda, S. and Hashimoto, S. (2006b). J. Photopolymer Sci. Tech. 19, 441-444.

Yoshida, R. (2008). Bull. Chem. Soc. Jpn 81, 676-688.

Yoshida, R., Sakai, T., Hara, Y., Maeda, S., Hashimoto, S., Suzuki, D. and Murase, Y. J. Controlled Release (2009). 140, 186-193.

Yoshida, R. (2010). Adv. Mater. 22, 3463-3483. 


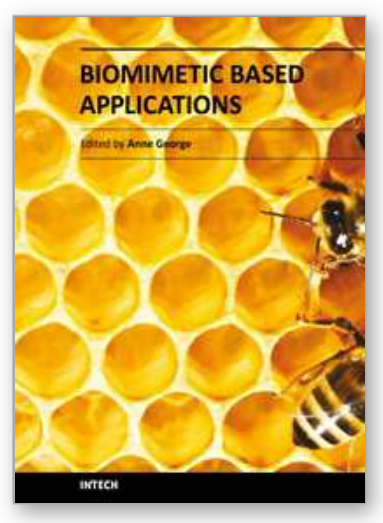

\author{
Biomimetic Based Applications \\ Edited by Prof. Marko Cavrak
}

ISBN 978-953-307-195-4

Hard cover, 572 pages

Publisher InTech

Published online 26, April, 2011

Published in print edition April, 2011

The interaction between cells, tissues and biomaterial surfaces are the highlights of the book "Biomimetic Based Applications". In this regard the effect of nanostructures and nanotopographies and their effect on the development of a new generation of biomaterials including advanced multifunctional scaffolds for tissue engineering are discussed. The 2 volumes contain articles that cover a wide spectrum of subject matter such as different aspects of the development of scaffolds and coatings with enhanced performance and bioactivity, including investigations of material surface-cell interactions.

\title{
How to reference
}

In order to correctly reference this scholarly work, feel free to copy and paste the following:

Ryo Yoshida (2011). Self-Oscillating Gel as Novel Biomimetic Materials, Biomimetic Based Applications, Prof. Marko Cavrak (Ed.), ISBN: 978-953-307-195-4, InTech, Available from:

http://www.intechopen.com/books/biomimetic-based-applications/self-oscillating-gel-as-novel-biomimeticmaterials

\section{INTECH}

open science | open minds

\section{InTech Europe}

University Campus STeP Ri

Slavka Krautzeka 83/A

51000 Rijeka, Croatia

Phone: +385 (51) 770447

Fax: +385 (51) 686166

www.intechopen.com

\section{InTech China}

Unit 405, Office Block, Hotel Equatorial Shanghai

No.65, Yan An Road (West), Shanghai, 200040, China

中国上海市延安西路65号上海国际贵都大饭店办公楼 405 单元

Phone: +86-21-62489820

Fax: +86-21-62489821 
(C) 2011 The Author(s). Licensee IntechOpen. This chapter is distributed under the terms of the Creative Commons Attribution-NonCommercialShareAlike-3.0 License, which permits use, distribution and reproduction for non-commercial purposes, provided the original is properly cited and derivative works building on this content are distributed under the same license. 